\section{\#P-BR-040}

BASIC RESEARCH

\title{
Influence of bone defect shape in implant surface decontamination using a protocol of spraying by high pressure water associated or not to sodium bicarbonate: an in vitro study
}

\author{
Dias, ECLCM; Nascimento, SFG; Napimoga, M; Capato, F
}

\section{Abstract}

The aim of this study was to evaluate the influence of bone defect shape in the efficacy of a high-pressure water spray protocol associated or not to sodium bicarbonate to decontaminate surfaces of dental implants.

Forty-eight titanium implants were distributed into four groups with different simulated defects: ten implants inserted into resin blocks with $20^{\circ}$ angle and $5 \mathrm{~mm}$ depth defects, sprayed with high-pressure water associated with sodium bicarbonate, ten implants inserted in resin blocks with $20^{\circ}$ angle and $5 \mathrm{~mm}$ depth defects, sprayed with high-pressure water not associated with sodium bicarbonate, ten implants inserted in blocks of resin with $50^{\circ}$ angle and $5 \mathrm{~mm}$ depth defects, sprayed with water in high-pressure associated with sodium bicarbonate and ten implants inserted in resin sites with $50^{\circ}$ angle and $5 \mathrm{~mm}$ depth defects, sprayed with highpressure water not associated with sodium bicarbonate. The implants were contaminated with Streptococcus mutans and after 72 hours they were submitted to the decontamination protocol.

It was verified that in the presence of defects of $20^{\circ}$ there was no statistically significant difference in bacterial quantification if water under pressure or water associated with sodium bicarbonate was used. In defects of $50^{\circ}$, there was a statistically significant reduction in the amount of bacteria when using water under pressure associated with sodium bicarbonate.

\section{Background and Aim}

Implant surface, when exposed to the buccal environment, undergoes several actions. One is the accumulation of bacterial biofilm. This triggers an inflammatory reaction with great potential for bone destruction, which may lead to peri-implant bone loss.

The aim of this study was to evaluate whether the different bone defect formats would influence the efficacy of a highpressure water spray protocol associated or not to sodium bicarbonate to decontaminate surfaces of dental implants installed in resin blocks with two different defect formats.

\section{Methods and Materials}

Forty-eight titanium implants were distributed into four groups with different simulated defects: ten implants inserted into resin blocks with $20^{\circ}$ angle and $5 \mathrm{~mm}$ depth defects, sprayed with high-pressure water associated with sodium bicarbonate, ten implants inserted in resin blocks with $20^{\circ}$ angle and $5 \mathrm{~mm}$ depth defects, sprayed with high-pressure water not associated with sodium bicarbonate, ten implants inserted in blocks of resin with $50^{\circ}$ angle and $5 \mathrm{~mm}$ depth defects, sprayed with water in high-pressure associated with sodium bicarbonate and ten implants inserted in resin sites with $50^{\circ}$ angle and $5 \mathrm{~mm}$ depth defects, sprayed with high-pressure water not associated with sodium bicarbonate. The implants were contaminated with Streptococcus mutans bacteria and after 72 hours they were submitted to the decontamination protocol. For each group, one implant were used as positive control and one implant as negative control.

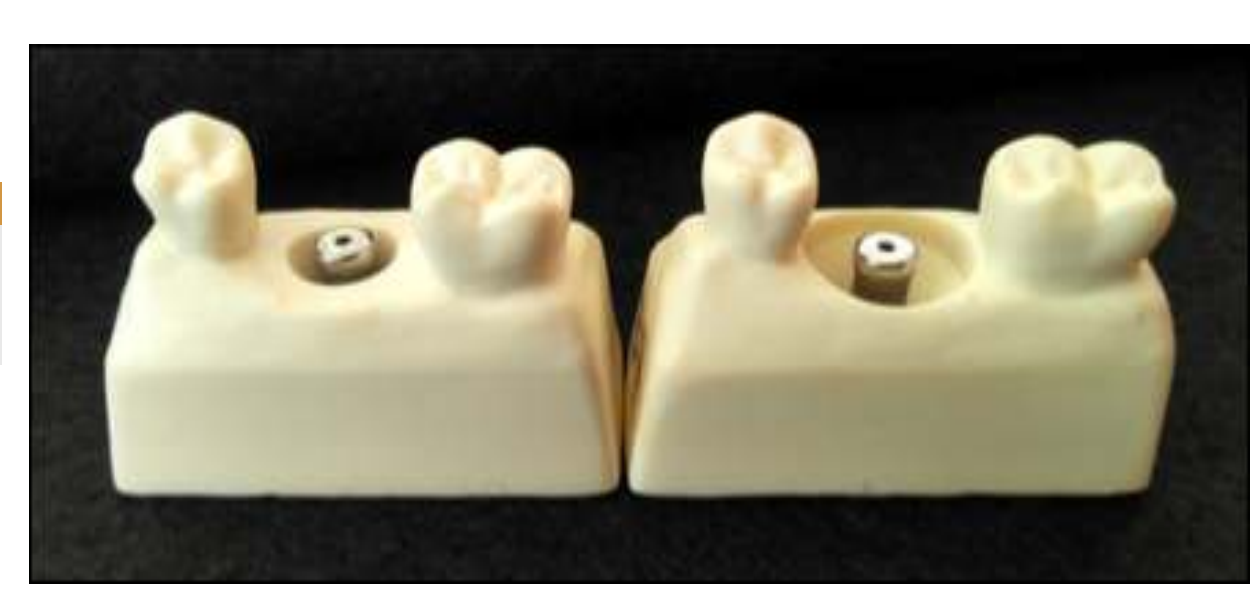

Fig 1. Resin block with different bone defects

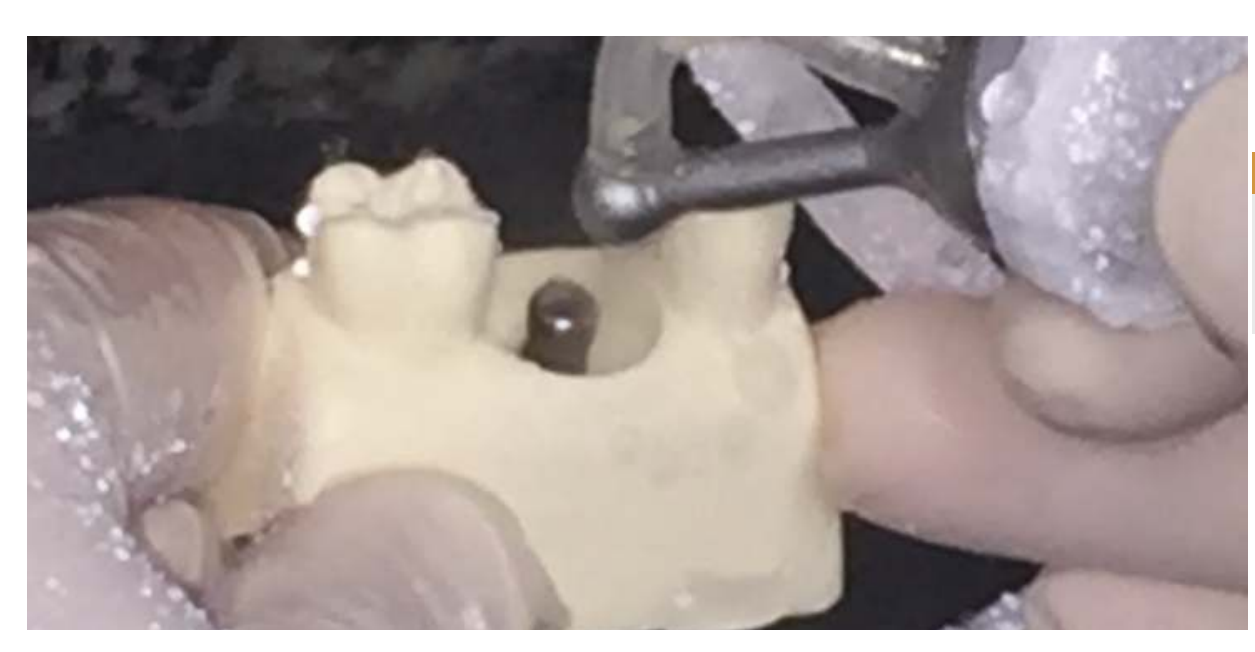

Fig 2. Implant surface decontamination with highpressure water associated with sodium bicarbonate
After microbiological tests, it was verified that in the presence of $20^{\circ}$ defects there was no statistically significant difference in bacterial quantification comparing water under pressure or water associated with sodium bicarbonate. In $50^{\circ}$ defects, there was a statistically significant reduction in the amount of bacteria when using high-pressure water associated with sodium bicarbonate.

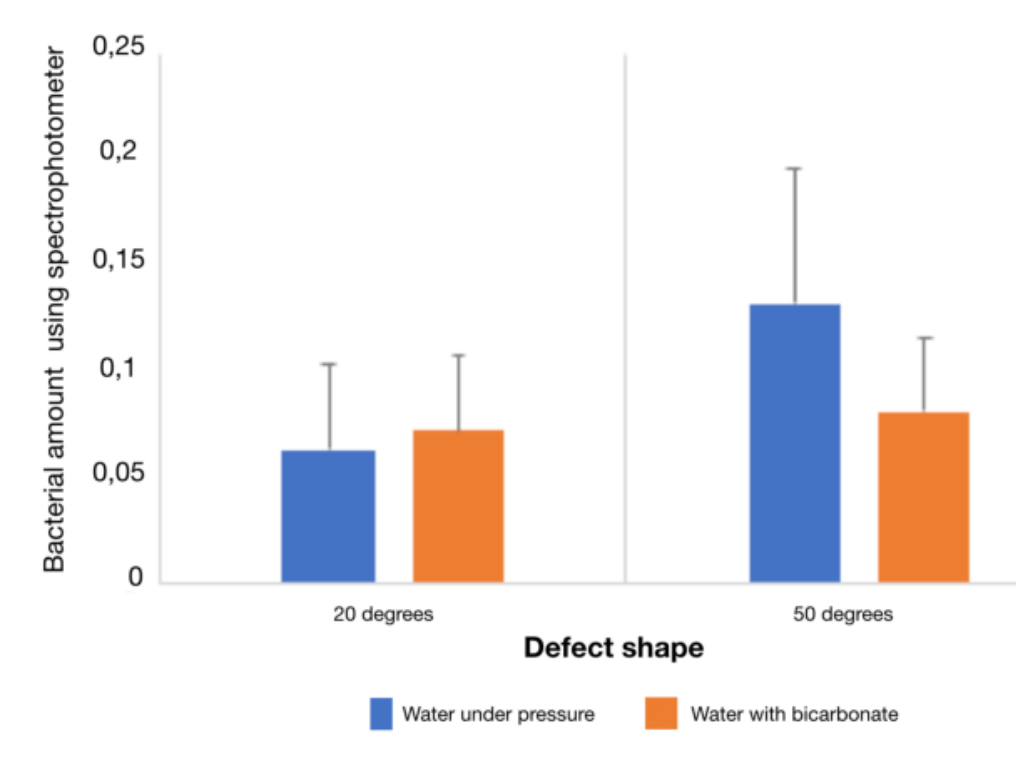

\section{Conclusion}

It can be stated that the types of defects did not influence the effectiveness of the decontamination protocol. However, the high-pressure water spray associated to sodium bicarbonate was more effective than spraying using high-pressure water alone.

Thus, the described decontamination protocol is effective on the removal of the bacterial biofilm adhered to the surface of the implants and can be used in the maintenance phase of the implants.

\section{References}

- Nemer Vieira LF, Dias ECLCM, Cardoso ES, Machado SJ, Pereira da Silva C, Vidigal GM Jr. Effectiveness of implant surfasse decontamination using a high-pressure sodium bicarbonate protocol: an in vitro study. Implant Dent. 2012 Oct;21(5):390-93.

2 - Valente NA, Andreana S. Peri-implant disease: what we know and what we need to know. J Periodontol Implant Sci. 2016 Jun;46(3): 136-51.

3 - Chambrone L, Wang HL, Romanos GE. Antimicrobial photodynamic therapy for the treatment of periodontitis and periimplantitis: An American Academy of Periodontology best evidence review. J Periodontal. 2018 Jul;89(7):783-803. 